\title{
РЕЗЕРВЫ ПОВЫШЕНИЯ ПРОИЗВОДИТЕЛЬНОСТИ ПОДСИСТЕМЫ «КАРЬЕРНЫЙ ТРАНСПОРТ - ДРОБИЛЬНЫЙ ПЕРЕДЕЛ" С УЧЕТОМ КОЭФФИЦИЕНТОВ ИСПОЛЬЗОВАНИЯ ДРОБИЛЬНОГО ОБОРУДОВАНИЯ
}

\author{
Журавлев Артем Геннадиевич1, \\ juravlev@igduran.ru \\ Черепанов Владимир Александрович1, \\ transport@igduran.ru \\ 1 Институт горного дела УрО РАН, \\ Россия, 620219, г. Екатеринбург, ул. Мамина-Сибиряка, 58.
}

\begin{abstract}
Актуальность. В современных условиях, когда необходимо наращивание производственной мощности действующих горнообогатительных комбинатов, повышение производительности карьера не может рассматриваться в отрыве от возможностей дробильно-обогатительных фабрик. При решении вопросов повышения мощности карьерного транспорта требуется уделять внимание оптимизации его взаимодействия с первичными стадиями обогатительного производства, а для этого необходимо рассматривать его совместно со звеном магистрального транспорта, как единую технологическую систему. Целью исследования является выявление резервов повышения производительности подсистемы «карьерный транспорт дробильный передел» с учетом коэфффициентов использования дробильного оборудования на основе обобщения опыта его эксплуатации и предъявляемых требований к эксплуатационной надежности, сложившихся в современных условиях.

Объекты: карьерный транспорт и дробильное оборудование.

Методы: хронометраж, обработка статистических данных, систематизация, анализ и обобщение, расчет производительности.

Результаты. Структурированы наиболее распространенные технологические схемы транспорта и дробильного оборудования, применяемые на современных ГОКах. Определены ключевые ограничивающие фракторы увеличения производительности для подсистемы горного предприятия «карьерный транспорт - дробильный передел». Произведены расчеты достижимой интенсивности загрузки различным карьерным транспортом для стабильной высокопроизводительной работы дробилок. Oпределены и рассчитаны показатели надежности работы дробилок. Выявлены основные факторы, влияющие на их эксплуатационную надежность, потребную периодичность и объем ремонтных работ, а также время производительной работы. Проведен анализ опьта эксплуатации дробилок, и сфрормулированы требования к организации их работы и порядка загрузки для обеспечения высокой производительности. Показано, что в условиях необходимости наращивания производственной мощности действующих ГОКов важным и реализуемым является использование резервов повышения производительности действующего дробильного оборудования: 10-30 \% за счет обеспечения равномерной подачи транспорта на разгрузку, 5-10 \% за счет оптимизации порядка работы цепи аппаратов дробильно-обогатительной фрабрики во времени, 3-5 \% за счет совершенствования технического обслуживания и ремонта. При этом повышение коэфффициента использования оборудования дробильнообогатительных фабрик необходимо рассматривать в увязке с работой технологического транспорта. Для стабильной высокопроизводительной работы транспортно-обогатительного передела горного предприятия необходимо одновременно налаживать организацию взаимодействия транспортного и дробильного оборудования и фрормировать эфрфективную систему обеспечения работоспособности этого оборудования, в том числе за счет максимального использования технических возможностей.
\end{abstract}

\section{Ключевые слова:}

Карьерный транспорт, дробилка, приемный бункер, коэфффициент готовности, эффективность, производительность, дробильное оборудование.

\section{Введение}

Технологические комплексы добычи и переработки руды являются взаимосвязанными системами и для рудных горнодобывающих предприятий и, как правило, объединены в горно-обогатительные комбинаты. Связующее звено между ними - технологический транспорт. Дробильно-обогатительные фабрики (ДОФ) размещены на различном расстоянии от карьера (группы карьеров), поэтому часто доставку руды осуществляет магистральное звено карьерного транспорта (железнодорожный, конвейерный, автомобильный).

В современных условиях, когда необходимо наращивание производственной мощности действующих ГОКов, повышение производительности карьера не может рассматриваться в отрыве от возможностей ДОФ. И если разработка карьера как постоянно видоизменя- ющейся выработки может быть относительно просто форсирована (в т. ч. за счет внедрения более высокопроизводительного оборудования), то для ДОФ рациональным является поиск резервов в рамках существующих производственных сооружений, с учетом массогабаритных характеристик эксплуатирующихся машин.

Среди прочих мероприятий важное значение имеет повышение коэффициента использования оборудования ДОФ, которое необходимо рассматривать в увязке с работой технологического транспорта. Характерным примером является повышение производительности головных дробилок крупного дробления, от которых зависит дальнейшая работа всей цепи аппаратов фабрики. Обеспечение их надежной и безаварийной работы одновременно с максимально возможной равномерностью загрузки позволяет повы- 
сить среднегодовую производительность даже без увеличения производительности (форсирования) самого оборудования. В случае наращивания мощности оборудования указанные мероприятия являются обязательными, поскольку в условиях плохо настроенной системы технической эксплуатации потери производительности будут еще больше.

Таким образом, при решении вопросов повышения мощности карьерного транспорта требуется уделять внимание оптимизации его взаимодействия с первичными стадиями обогатительного производства. Для этого необходимо рассматривать обогатительное производство совместно со звеном магистрального транспорта как единую технологическую систему.

Наиболее распространены следующие технологические схемы транспорта (табл. 1):

- автомобильный сборочно-магистральный (при относительно небольших объемах до 10-15 млн т/год либо высокой динамике продвижения горных работ, делающей затратным постоянный перенос железнодорожных путей и нецелесообразным размещение конвейерного подъемника);

- автомобильно-железнодорожный (самый массовый на крупных ГОКах);

- автомобильно-конвейерный (для карьеров, отрабатываемых по углубочной системе разработки [1]), когда дробленая руда может подаваться непосредственно на стадию среднего и мелкого дробления;

- автомобильно-конвейерно-железнодорожный (формируется при внедрении в существующий автомобильно-железнодорожный транспорт конвейерного звена (циклично-поточной технологии) с переносом железнодорожного звена за пределы карьера) рационален при значительной удаленности ДОФ от карьера.

Практически все описанные схемы транспорта идентичны по параметрам и конструкции приемных устройств дробильного-обогатительных фабрик:

- при относительно невысокой производительности применяются щековые дробилки, загружаемые из приемного бункера питателем;

- при значительных объемах (более 10-15 млн т/год) используются конусные дробилки с непосредственной загрузкой из приемного бункера.

В последнее десятилетие в России получают распространение схемы с автомобильно-автомобильным транспортом, когда магистральные перевозки осуществляются автопоездами. Такой вид транспорта обеспечивает существенное снижение себестоимости перевозок по сравнению с карьерными автосамосвалами и менее требователен в вопросах инфраструктуры в сравнении с железнодорожным транспортом [2-4]. В этом случае приемная способность участка крупного дробления зависит от организации работ по перевалке руды со склада в приемный бункер (погрузчиком при малом расстоянии либо автомобильным транспортом с удаленного склада).

Таким образом, в рассматриваемой постановке задачи производительность карьера и транспорта огра- ничивается производительностью системы «разгружаемый транспорт - приемный бункер - дробилка крупного дробления - смежное оборудование» либо «подающий конвейер - дробилки среднего и мелкого дробления - смежное оборудование». Рассмотрим пределы возможной оптимизации указанных систем.

Ключевыми ограничивающими факторами для системы являются:

- интенсивность и равномерность подачи транспорта на разгрузку;

- форма и вместимость приемного бункера, количество мест для одновременной разгрузки;

- вместимость транспортных единиц (автосамосвалов, думпкаров);

- часовая производительность дробилки с учетом свойств руд (прочности и абразивности и др.), а также их гранулометрического состава;

- возможность работы дробилки крупного дробления под завалом;

- надежность дробилки, продолжительность простоев в ремонте с учетом свойств руд (прочности и абразивности и др.);

- производительность смежного оборудования (питатели, конвейеры, грохоты);

- надежность и трудоемкость ремонтного обслуживания смежного оборудования.

\section{Методы исследования}

Исследование выполнено с использованием данных об эксплуатации щековых дробилок с простым качанием щеки, а также конусных дробилок крупного, среднего и мелкого дробления, производимых ПАО «Уралмашзавод». Для формирования статистической базы данных собрана информация с действующих горнодобывающих предприятий: Ковдорский ГОК (г. Ковдор), Учалинский ГОК (г. Учалы), Северский карьер (г. Екатеринбург, п. Северка), Сибирский карьер (г. Екатеринбург), ОАО «Ураласбест» (г. Асбест), Михайловский ГОК (г. Железногорск), Лебединский ГОК (г. Губкин), Стойленский ГОК (г. Старый Оскол), Гайский ГОК (г. Гай), АО «Апатит» (г. Кировск), Магнитогорский металлургический комбинат (г. Магнитогорск), Качканарский ГОК (г. Качканар), АО «Карельский окатыш» (г. Костомукша), РУПП «Гранит (г. Микашевичи, Республика Беларусь), Южный ГОК, Центральный ГОК, Ингулецкий ГОК (г. Кривой Рог, Украина).

Выполнялся: хронометраж разгрузочных операций, хронометраж операций по техническому обслуживанию и ремонту, обработка статистических данных о режимах работы и простоях оборудования в ремонте.

Производительность дробилок определена расчетным методом с учетом свойств дробимых руд, формы и конструкции приемных устройств, вида и конструктивных особенностей транспорта, технических характеристик дробилок.

Систематизация условий эксплуатации дробилок по свойствам дробимых пород и режимам работы выполнена на основе статистической обработки путем анализа и обобщения 
Таблица 1. Наиболее распространенные технологические схемы транспорта и приемные устройства дробильно-обогатительной фабрики

Table 1. The most common technological schemes for quarry transport and receiving devices for crushing and processing mills

Технологическая схема транспорта/Technological scheme of transport

\section{Автомобильно-}

железнодорожный

транспорт

Road and rail transport

Автомобильно-

конвейерный транспор (т. н. циклично-поточная

технология - ЦПТ)

Auto-conveyor transpo

(in-pit crushing and

conveying technology -

CCT)

конвейерно-железнодо-

рожный (как правило

после внедрения ЦПТ в

Auto-conveyor-rail (in
most cases, after the

introduction of the $\mathrm{CCT}$

in the existing auto-

railway transport)

Автомобильно-

автомобильный

Auto-Auto

Сборочный автомобильный транспорт (карьерные автосамовалы) от забоев до ПП.

2) Магистральный железнодорожный транспорт (составы:

яговый агрегат + думпкары) от ПП в карьере до ДОФ

1) Assembly road transport (mining dump trucks) from the faces to the RP.

Mains railway transport (trains: traction unit + dumpcars) from

RP in the quarry to the crushing and processing mills (CPM)

Сборочный автомобильный транспорт (карьерные автосамо-

ДОФ на стадию среднего дробления

to the CRS.

2) The mains conveyor transport from the CRS in the quarry to the $\mathrm{CPM}$ at the standard cone crusher

Сборочный автомобильный транспорт (карьерные автосамосвалы) от забоев до ДПП.

Промежуточный магистральный конвейерный транспорт о ДПП в карьере до ПП на борту карьера.

Магистральный железнодорожный транспорт (составы:

ұяговый агрегат + думпкары) от ПП на борту карьера до ДОФ

Gatom

Производитель-

\begin{tabular}{l} 
Hость, МлН Т/ГО \\
Productivity \\
\hline
\end{tabular}

Productivity
million tons/yea

\begin{tabular}{l|l}
$<10-15$ & Приемный бункер с питателем, подающим руду на щековые дробилки
\end{tabular}

Receiving bin with feeder feeding ore to jaw crushers

\begin{tabular}{l|l}
$>10-15$ & Приемный бункер с непосредственно установленной конусной дробилкой ККД
\end{tabular}

\begin{tabular}{l|l|l}
$<10-15$ & Сборочный & Приемный бункер с питателем, подающим руду на щековые дробилки
\end{tabular} автотранспорт $\quad$ Receiving bin with feeder feeding ore to jaw crushers

Gathering road transport Приемный бункер с непосредственно установленной конусной дробилкой ККД

(mining dump trucks) $\quad$ Receiving bin with directly mounted coarse crusher

\begin{tabular}{l|l|l}
$>10-15$ & Магистральный кон- & На стадию среднего и мелкого дробления (напрямую или через усреднительный
\end{tabular} вейер склад)

The mains conveyor At the stage of medium and fine crushing (directly or through an coarse/run of mine transport stockpile)

Приемный бункер стадии крупного дробления

Receiving bin of coarse crushing stages Receiving bin of stage medium or fine crushing

$>10-15$

Ithe CRS in the

quarry to the RP on board the quarry.

Mains railway transport (trains: traction unit + dumpcars) from

the RP on board the quarry to the CPM

1) Сборочный автомобильный транспорт (карьерные автосамо-

свалы) от забоев до ПП на поверхности

Магистральные автопоезда от ПП до ДОФ

Gathering road transport (mining dump trucks) from the bottom

Mains road trains from RP to CPM

П) Пборочный автомобильный транспорт лы на крутонаклонных съездах

Специальные виды карьерного транспорта (скиповые подъемни-

ки, ГПКД и др.)

Special types of career

transport (skip hoists,

FC, etc.)

2) Промежуточный магистральный подъемник (скип, автомобильный, ГПКД).

3) Поверхностный магистральный транспорт (железнодорожный, автопоезда)

1) a) Gathering road transport

dump trucks at steep ramp

Intermediate main elevator (skip, automobile, FC)

3) Surface mains transport (railway, road train) реклаймером) на среднее дробление

for medium crushing

а) Разгрузка на «почву» рудного склада ДОФ или со специаль-

$\begin{array}{ll}<7-10 & \text { б) Разгрузка в приемный бункер ДОФ }\end{array}$

a) Unloading at the bottom of the ore storage of the CPM or from a special flyover. Overload by loaders in the Receiving bin of CPM. b) Unloading into the bunker CPM

а) Разгрузка на «почву» рудного склада ДОФ или со специальной эстакады. Перегрузка погрузчиками в бункер ДОФ.

)

(he CPM or from a

.

b) Unloading into the bunker $\mathrm{CPM}$ ной эстакады. Перегрузка погрузчиками в бункер ДОФ.
Бункер с конусной или щековой дро-

Receiving bin with cone or jaw crushe

Бункер с питателем.

болача конвером на среднее дробление

iving bin with feeder.

Conveyor feed for medium crushing

Приемный бункер с питателем, подающим руду на щековые дробилки

Receiving bin with feeder feeding ore to aw crushers

Приемный бункер с установленной ККД Receiving bin with coarse crusher Приемный бункер с питателем, подающим руду на щековые дробилки jaw crushers

Приеннй бункер с установленной ККд Receiving bin with coarse crusher

$\bullet$ ДПП-дробильно-перегрузочный пункт, ППП - перегрузочный пункт; ГПКД - грузовая подвесная канатная дорога.

$C R S$ - crushing and reloading station; $R P$ - reloading point; $F C$ - freight cableway. 


\section{Результаты исследования}

Схемы загрузки приемных устройств ДОФ приведены на рис. 1. Анализ показал, что предельная интенсивность загрузки дробилки крупного дробления с целью максимального использования ее производительности определяется количеством мест разгрузки, продолжительностью разгрузки одного транспортного средства (самосвал, думпкар, погрузчик), скоростью обмена транспортных средств, равномерностью подачи транспорта на разгрузку.

Расчеты достижимой интенсивности загрузки (рис. 2) показали, что возможностями обеспечения наиболее стабильного высокопроизводительного по-

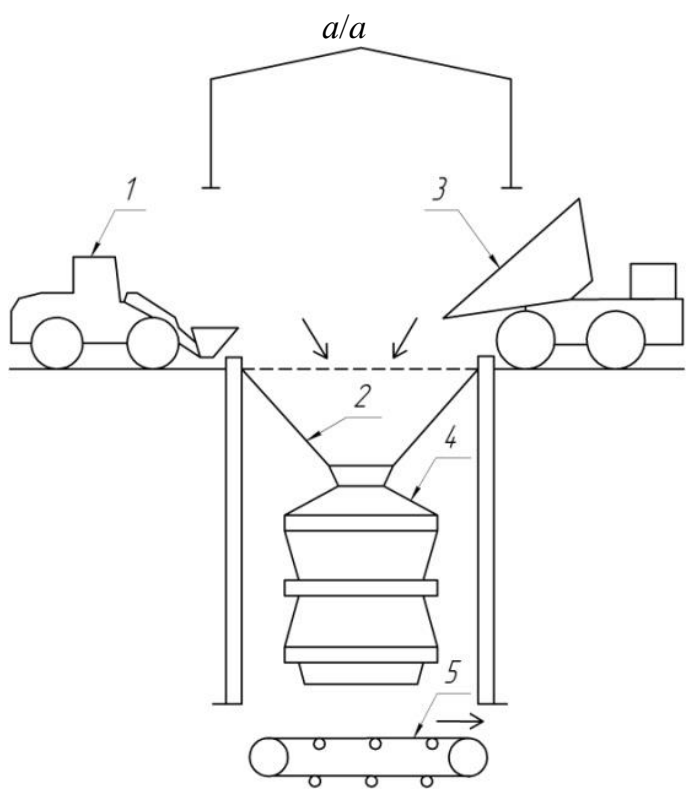

тока обладает автомобильный транспорт за счет высокой технологической «гибкости» и при этом значительной грузоподъемности (до 360 т).

На рис. 2 представлены зависимости производительности разных видов транспорта от их грузоподъемности с наложением производительности конусных дробилок разных модификаций (горизонтальные линии). На пересечении наклонных и горизонтальных линий определяется необходимая грузоподъемность. Неравномерность подачи транспорта на разгрузку уменьшает интенсивность ее загрузки, поэтому при коэффициенте неравномерности $K_{\mathrm{H}}<0,9$ она заметно снижается.
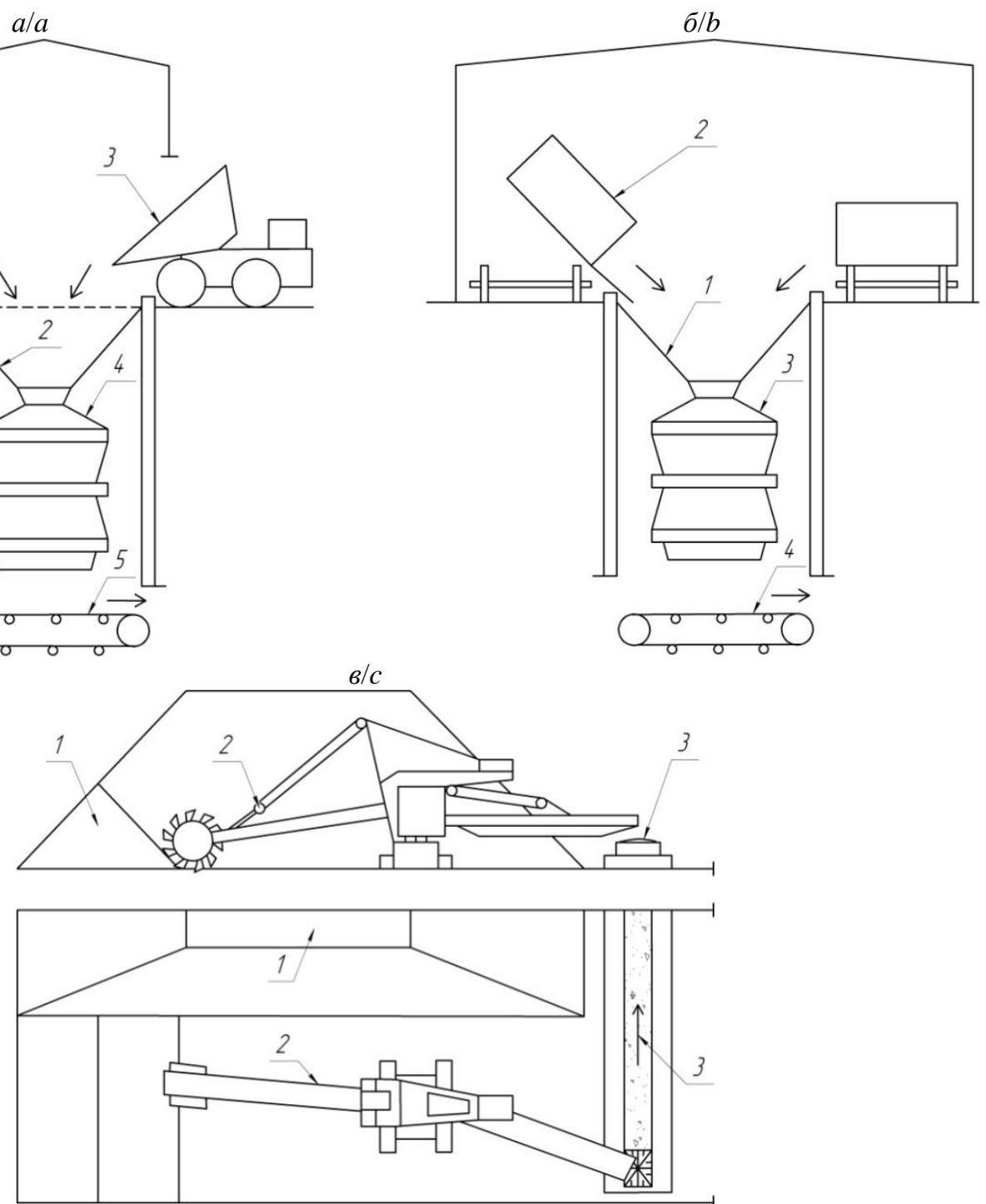

Pис. 1. Схемы приемных бункеров дробильно-обогатительных фабрик: а) разгрузка автомобильного транспорта 3 (погрузчика - 1) в приемный бункер - 2, на дробилку - 4 и далее по технологической цепочке аппаратов - 5 на ДОФ; б) разгрузка железнодорожного транспорта - 2 в приемный бункер - 1, на дробилку - 3 и далее по технологической цепочке аппаратов - 4 на ДОФ; в) отгрузка руды с усреднительного склада - 1 иепным экскаватором или реклаймером - 2 с отгрузкой на конвейер - 3, доставляющий руду до ДОФ

Fig. 1. Schemes of receiving bins of crushing and processing plants: a) unloading quarry automobile transport - 3 (loader1) into the receiving bin -2 , to the crusher -4 and then along the technological chain of devices -5 to the crushing and processing plants; b) unloading railway transport -2 into the receiving bin -1 , to the crusher -3 and then along the technological chain of vehicles -4 to the crushing and processing plants; $c$ ) unloading from the averaging ore storage -1 with a chain excavator or reclaimer -2 with unloading to the conveyor -3 , which delivers the ore to the crushing and processing plants 
Погрузчики при перевалке руды из рудного склада вблизи приемного бункера ДОФ также могут обеспечить высокую стабильность потока, но обладают меньшей производительностью, а потому для загрузки современных форсированных дробилок, например, ККД-1500, как правило, требуется несколько (2-3 шт.) погрузчиков.

При высокой производительности железнодорожного транспорта, измеряемой в грузообороте (т·км), интенсивность его разгрузки ограничивается инертностью за счет увязанности транспортных сосудов (думпкаров) в единый состав, наличием ручного управления разгрузкой, увеличенным (в сравнении с автомобильным транспортом) временем обмена транспорта у приемного бункера. В связи с этим он занимает среднее положение по интенсивности загрузки приемного бункера дробилки (рис. 2).

Отметим, что для интенсивной загрузки современной форсированной дробилки (производительность 5000-6000 т/ч) при одном разгрузочном месте у бункера требуются автосамосвалы высокой грузоподъемности (240-360 т). Поэтому целесообразно использование схем с двумя местами для одновременной разгрузки, что позволит обеспечить требуемый уровень даже при высокой неравномерности $\left(\mathrm{K}_{\mathrm{H}}=0,7\right.$ и ниже) подачи автосамосвалов грузоподъемностью 90-130 т. Эта же схема дает перспективу для загрузки форсированных дробилок с производительностью 7000-7500 т/ч автосамосвалами грузоподъемностью 130-180 т, в зависимости от равномерности работы автотранспорта.

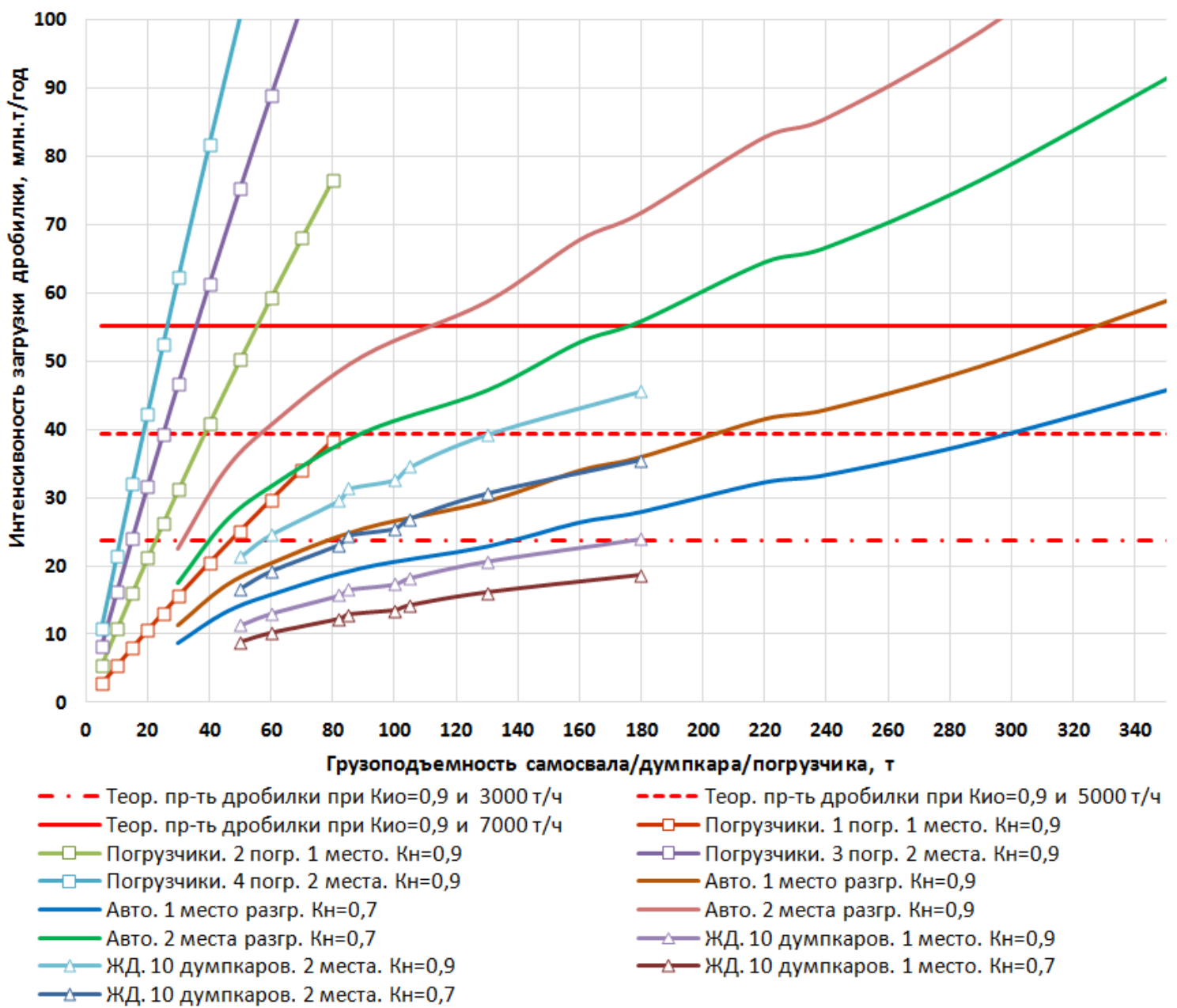

Pис. 2. Достижсимая интенсивность загрузки приемного бункера (Кн-коэффициент равномерности подачи транспорта, равный отношению времени наличия транспорта на разгрузке к суммарному времени готовности дробилки к загрузке)

Fig. 2. Reachable receiving bin loading intensity (Kn - the coefficient of evenness of transport supply equal to the ratio of transport presence time at unloading to the total time of the crusher readiness for loading)

Для железнодорожного транспорта не исчерпаны возможности уже ставшей классической схемы с двусторонней загрузкой бункера. При этом для форсированных дробилок (5000-6000 т/ч) необходимо применение думпкаров повышенной грузоподъемности (160-180 т) при обеспечении большей равномерности подачи составов на разгрузку $\left(K_{\mathrm{H}}=0,87-0,95\right)$ за счет технологических мероприятий и совершенствования схемы путевого развития. Схемы с одним местом разгрузки подходят для щековых дробилок при годовой производительности до 14 млн т/год.

Вторая группа из наиболее значимых факторов, определяющих производительность системы «транспорт - приемный бункер - дробилка крупного дробле- 
ния - смежное оборудование», относится к надежности работы дробилки и смежного с ней оборудования.

Анализ надежности необходим для выявления узких мест в системе и для поиска компонентов или подсистем с низкой надежностью для заданной проектной производительности, а также полезен для определения интервалов планирования и организации технического обслуживания [5, 6]. Использование информации об эксплуатационной надежности и готовности к работе оборудования позволяет максимизировать общую производительность системы [7].

Исследования показателей наработки узлов и деталей конусных и щековых дробилок на горнодобывающих предприятиях позволили выявить основные факторы, влияющие на их эксплуатационную надежность, потребную периодичность, объем ремонтных работ и время производительной работы:

1) Крепость перерабатываемого материала. Высокая прочность вызывает значительные нагрузки в узлах трения, повышенные нагрузки на корпусные детали, повышенный износ броней (чаще в виде трещин, местного сквозного износа).

2) Абразивность перерабатываемого материала. Характеризует способность горных пород изнашивать рабочие органы в процессе дробления (измельчения) при трении. Например, в случае относительно непрочных, но высокоабразивных пород, брони дробилки будут изнашиваться практически равномерно по толщине (при правильной загрузке дробилки), характер износа - истирание.

3) Крупность кусков питания и степень дробления. Влияют на нагрузки в узлах и механизмах, а увеличение данных показателей ведет к повышению потребляемой мощности. Наличие большого количества кусков, близких к максимальной крупности питания, существенно увеличивает нагрузку на привод и корпус дробилки.

4) Объем перерабатываемой горной массы. Производительность напрямую влияет на износ и расход ресурса всех узлов дробилки.

5) Попадание недробимого тела в зону дробления. $\mathrm{He}$ допускается, поскольку создает ударные нагрузки и, как правило, вызывает повреждение элементов дробилки.

6) Соблюдение периодичности и качество выполнения ТОиР, качество применяемых запасных частей и материалов, своевременность выявления зарождающихся неисправностей и предотвращение их развития до ближайшего ремонта либо устранение. Влияет на износ и расход ресурса всех узлов дробилки. Вероятность отказа оборудования зависит от его качества, а также от условий эксплуатации и эффективности обслуживания Поддержание оборудования в нормальном рабочем состоянии и сохранение необходимой производительности требует надлежащего технического обслуживания в течение всего жизненного цикла всего оборудования, входящего в одну систему [8] Для эффективной эксплуатации необходимо сокращать время простоя оборудования, повышать его надежность и ремонтопригодность [9-12].
Интегральным показателем, отражающим надежность работы дробилки во времени, простои в техническом обслуживании и ремонтах (ТОиР), а значит ее производительность, является коэффициент готовности $[13,14]$.

В общем случае, согласно ГОСТ 27.002 [15], различают коэффициент внутренней готовности, определяемый при проектировании для расчетных условий эксплуатации, технического обслуживания и ремонта, и коэффиичент эксплуатационной готовноcmu, определяемый для реальных условий эксплуатации, технического обслуживания и ремонта по фактическим показателям. Применительно к дробилкам термины «коэффициент технической готовности дробилки» и «коэффициент внутренней готовности дробилки» предлагается определять следующим образом.

Коэффициент внутренней готовности дробилки:

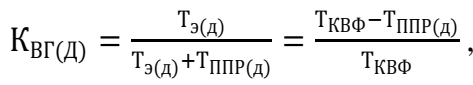

где $\mathrm{T}_{\text {эд) }}$ - суммарное время пребывания дробилки в работоспособном состоянии, в рассматриваемом

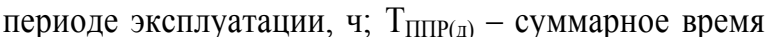
пребывания дробилки в неработоспособном состоянии в связи с ее планово-предупредительными техническими обслуживаниями и ремонтами, заложенными в технологическом регламенте (производителя оборудования или эксплуатирующего предприятия), ч; $\mathrm{T}_{\text {КФв }}$ - календарный фонд времени за рассматриваемый период, ч.

Коэффициент технической готовности дробилки - это отношение суммарного времени пребывания дробилки в работоспособном состоянии за некоторый период эксплуатации к суммарному времени пребывания дробилки в работоспособном состоянии и простоев, обусловленных техническим обслуживанием и ремонтом, за тот же период:

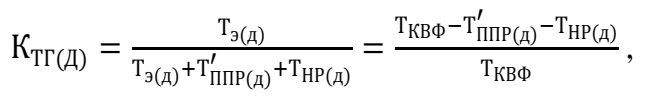

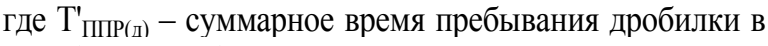
неработоспособном состоянии в связи с ее плановопредупредительными техническими обслуживаниями и ремонтами, предусмотренными по графику предприятия, эксплуатирующего дробилку, в рассматриваемом периоде эксплуатации, ч; $\mathrm{T}_{\mathrm{HP}(д)}-$ суммарное время пребывания дробилки в неработоспособном состоянии в связи с простоями из-за внеплановых ее ремонтов в рассматриваемом периоде эксплуатации, ч.

Коэффициент технической готовности комплекса «дробилка + смежное оборудование» можно определить по формуле:

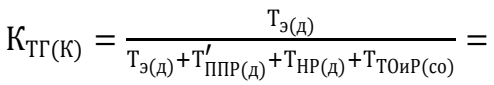

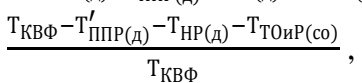

где $\mathrm{T}_{\mathrm{TOиP}}(\mathrm{co)}$ - суммарное время пребывания смежного оборудования в неработоспособном состоянии в связи с его планово-предупредительными, а также внеплановыми ремонтами, не совмещенными во времени с ТОиР дробилки, ч. 
На рис. 3 приведены графики зависимости достижимой годовой выработки (млн т/год) конусной дробилки крупного дробления ККД-1500/180 от коэффициента готовности $\left(K_{\mathrm{r}}\right)$ при разной степени форсирования дробилки (часовой производительности) и коэффициенте использования во времени (К ив).

Видно, что снижение коэффициента готовности дробилки на 5 \% (наклонные прямые графики) ведет к падению годовой производительности на 2-3 млн т.

Современные дробилки, например производимые ПАО «Уралмашзавод», обеспечивают значительно более высокую производительность в сравнении с их ранними модификациями 1980-х гг. выпуска (которые до настоящего времени продолжают эксплуатироваться на ряде горнодобывающих предприятий ввиду их большого ресурса). Так, для АО «Стойленский ГОК» спроектирована модификация, обеспечивающая производительность 6000 т/ч при крупности дробленого продукта 300 мм [16]. Поэтому рациональное использование производственной мощности форсированных дробилок является актуальной задачей, поскольку час простоя по технологическим или техническим причинам для более мощного оборудования обходится дороже.

Вертикальными линиями на рис. 3 показаны достижимые коэффициенты внутренней готовности $\left(K_{\text {вг }}\right)$ дробилки ККД-1500 производства ПАО «Уралмашзавод» в стандартных условиях $\left(K_{\text {вг(д) }}=0,98\right)$ и особо тяжелых условиях $\left(\mathrm{K}_{\mathrm{Br}(\mathrm{z})}=0,93\right)$ эксплуатации (характеристику условий см. в табл. 2). Они рассчитаны по данным «Регламента на техническое обслуживание и ре- монт», разработанного ИГД УрО РАН по заказу ПАО «Уралмашзавод» [17], и отражают теоретически достижимый уровень технической готовности дробилки и соответствующую ему годовую производительность.

Ряд факторов в реальных условиях эксплуатации обуславливают снижение коэффициента готовности в связи с внеплановыми остановками, ремонтами (они могут быть вызваны, например, попаданием недробимого тела или прохождением сверхгабаритного куска горной массы в питании и др.). В итоге на практике $K_{\text {тг(д) }}$ обычно характеризуется величиной 0,92 0,96 , в отдельных случаях снижаясь до 0,89 . Это приводит к падению годовой выработки на 1-2 млн т для нефорсированных дробилок (4000 т/ч) и на 2-4 млн т для форсированных (6000 т/ч).

Поскольку дробилки не могут выполнять технологические задачи сами по себе, они всегда включены в комплексы оборудования (как минимум непосредственно связаны с приемным бункером и разгрузочным конвейером, чаще через поддробильный бункер и питатель). Поэтому выполнение плановых и внеплановых ремонтов всего комплекса взаимосвязаны во времени. Это накладывает дополнительные затраты времени на ТОиР и, следовательно, снижает К тг(д). На рис. 3 этот диапазон коэффициентов готовности обозначен $\Delta \mathrm{K}_{\text {тг(к) }}\left(K_{\text {тг }}\right.$ комплекса). Практика горнодобывающих предприятий показывает, что $\mathrm{K}_{\text {тг(к) }}$ может составлять порядка $0,87-0,95$, а в отдельных случаях, ввиду технологических особенностей дробильного передела и низкого уровня организации технической

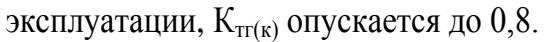

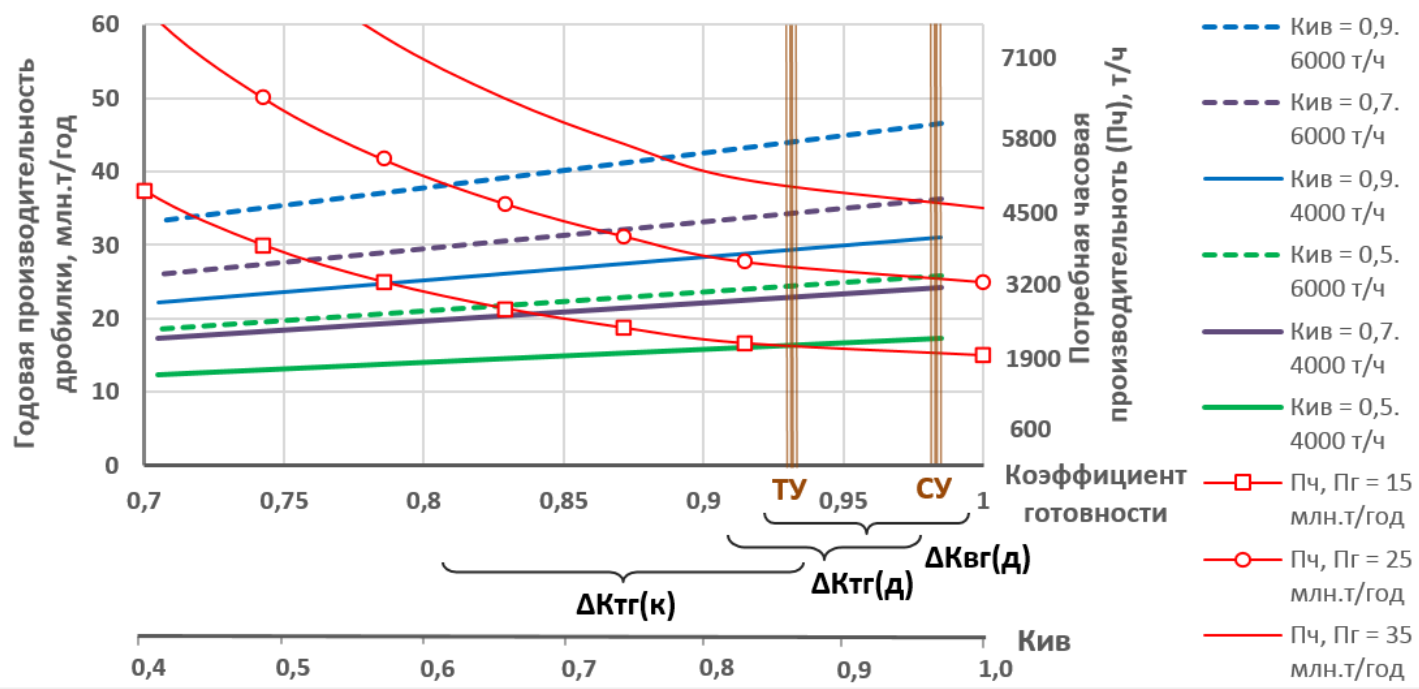

Рис. 3. Достижимая годовая производительность дробилки ККД-1500/180 ПАО «Уралмашзавод» (модификации с

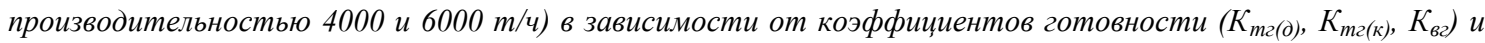
использования во времени $\left(K_{u в}\right)$, а также потребная часовая производительность $\left(\Pi_{4}\right)$ в зависимости от коэффициента использования во времени $\left(K_{\text {ив}}\right)$ при разной плановой годовой $\left(\Pi_{2}\right)$ производительности $($ ТУ -

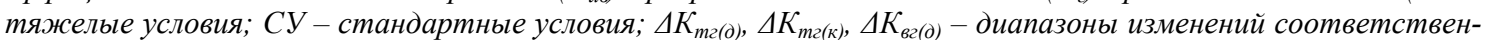
но: коэффициентов технической готовности дробилки и комплекса «дробилка + смежное оборудование», коэффициента внутренней готовности дробилки)

Fig. 3. Achievable annual capacity of the KKD-1500/180 crusher of «Uralmashplant» JSC (modifications with a capacity of 4000 and $6000 \mathrm{t} / \mathrm{h})$ depending on the availability and time-use factors $\left(K_{i v}\right)$, as well as the required hourly output $\left(P_{c h}\right)$, depending on the required annual $\left(P_{g}\right)$ capacity $\left(D C\right.$ - difficult conditions; $S C$ - standard conditions; $\Delta K_{t g(d)}$, $\Delta K_{t g(k)}, \Delta K_{v g(d)}$ - ranges of changes, respectively: technical readiness coefficients of the crusher and the "crusher + related equipment» complex, crusher internal readiness coefficient) 
Таблица 2. Характеристика условий дробления пород

Table 2. Description of rock crushing conditions

\begin{tabular}{|c|c|c|}
\hline $\begin{array}{l}\text { Характеристика дробимых пород } \\
\text { Description of crushed rocks }\end{array}$ & $\begin{array}{l}\text { Стандартные условия } \\
\text { Standard conditions }\end{array}$ & $\begin{array}{c}\text { Высокоабразивные крепкие руды } \\
\text { Highly abrasive strong ores }\end{array}$ \\
\hline Плотность в целике/Densities in the rear & $2-3 \mathrm{~T} / \mathrm{M}^{3}\left(\mathrm{t} / \mathrm{m}^{3}\right)$ & $\begin{array}{c}\text { до } 4 \mathrm{~T} / \mathrm{M}^{3} \\
\text { up to } 4 \mathrm{t} / \mathrm{m}^{3}\end{array}$ \\
\hline Насыпная плотность/Bulk density & $2 \mathrm{~T} / \mathrm{M}^{3}\left(\mathrm{t} / \mathrm{m}^{3}\right)$ & $\begin{array}{c}\text { до } 2,5 \mathrm{~T} / \mathrm{M}^{3} \\
\text { up to } 2,5 \mathrm{t} / \mathrm{m}^{3}\end{array}$ \\
\hline Влажность/Humidity & \multicolumn{2}{|c|}{ не выше $4 \% /$ not higher than $4 \%$} \\
\hline $\begin{array}{l}\text { Абразивность (Ai) по Бонду } \\
\text { Bond abrasiveness (Ai) }\end{array}$ & $\begin{array}{c}\text { средняя } \\
\text { (Ai не выше } 0,4 \text { г) } \\
\text { average } \\
\text { (Ai not higher than } 0,4 \mathrm{~g} \text { ) }\end{array}$ & $\begin{array}{c}\text { крайне высокая } \\
\text { (Ai=0,5-0,7 г и болеe) } \\
\text { extremely high } \\
\text { (Ai=0,5-0,7 g or more) }\end{array}$ \\
\hline $\begin{array}{c}\text { Предел прочности пород при одноосном } \\
\text { сжатии } \\
\text { Breaking strength under uniaxial compression }\end{array}$ & 100-110 Мпа (MPa) & $\begin{array}{c}\text { 140-170 МПа } \\
\text { Bap. II*: } 180-250 \mathrm{M \Pi а} \\
\text { 140-170 MPa } \\
\text { Variant II*: } 180-250 \mathrm{MPa}\end{array}$ \\
\hline Режим работы/Mode of operation & $\begin{array}{l}\mathrm{K}_{\text {ив }}=0,75-0,85 \\
\mathrm{~K}_{\mathrm{iv}}=0,75-0,85\end{array}$ & $\begin{array}{c}\mathrm{K}_{\text {ив }}=0,75-0,85 \\
\text { Bap. II*: } \mathrm{K}_{\text {ив }}=0,86-0,95 \\
\mathrm{~K}_{\mathrm{iv}}=0,75-0,85 \\
\text { Variant II*: } \mathrm{K}_{\mathrm{iv}}=0,86-0,95\end{array}$ \\
\hline $\begin{array}{l}\text { Крупность кусков } \\
\text { Sized rock }\end{array}$ & \multicolumn{2}{|c|}{$\begin{array}{l}\text { В пределах технической характеристики дробилки } \\
\text { Within the technical specifications of the crusher }\end{array}$} \\
\hline $\begin{array}{c}\text { Попадание недробимых тел } \\
\text { Hazardous bodies }\end{array}$ & \multicolumn{2}{|c|}{ не допускается, носит единичный характер/not allowed, is single } \\
\hline $\begin{array}{r}\text { Ремонт выполь } \\
\text { ПАО « } \\
\text { Repair is carried ou }\end{array}$ & $\begin{array}{c}\text { игинальными запасными г } \\
\text { завод», и рекомендованнь } \\
\text { ommended materials and or } \\
\text { JSC Uralmashzavod }\end{array}$ & $\begin{array}{l}\text { вляемыми } \\
\text { ми } \\
\text { rts supplied by }\end{array}$ \\
\hline
\end{tabular}

*Вар. II - наиболее тяжелье условия работы высокофорсированных дробилок.

*Variant II - the most difficult working conditions for high-performance crushers.

Существенное влияние на производительность оказывают организационно-технологические простои, характеризуемые коэффициентом использования дробильного оборудования во времени $\left(K_{\text {ив }}\right)$. По рис. 3 видно, что для поддержания заданной годовой производительности с уменьшением К кв требуется все больше форсировать дробилку, т. е. увеличивать ее производительность (графики красного цвета). Причем, если при $\mathrm{K}_{\text {ив }}=0,9-1,0$ зависимость близка к прямолинейной, то в диапазоне $\mathrm{K}_{\text {ив }}<0,9$ она изменяется возрастающими темпами (становится криволинейной).

В целом степень полезного использования дробилки характеризуется экстенсивным коэффициентом использования оборудования (часто его называют просто

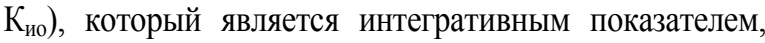
учитывающим все организационно-технологические и ремонтно-технические простои. Его можно выразить через описанные выше коэффициенты:

$$
\begin{aligned}
& \kappa_{\text {ио }}=K_{\text {тг (к) }} * K_{\text {ив }}=\left[K_{\text {тг(д) }}-\frac{\mathrm{T}_{\text {ТОиР(со) }}}{\mathrm{T}_{\text {КВФ }}}\right] * K_{\text {ив }}= \\
& =\left[\left(\mathrm{K}_{\text {вг(д) }}-\frac{\mathrm{T}_{\text {НР(д) }}}{\mathrm{T}_{\text {КВ }}}\right)-\frac{\mathrm{T}_{\text {ТонР }((0)}}{\mathrm{T}_{\text {КВ }}}\right] * \mathrm{~K}_{\text {ив }},
\end{aligned}
$$

где $K_{\text {ив }}$ - коэффициент использования оборудования во времени, равный отношению времени работы оборудования к общему времени нахождения в готовом к работе состоянии (т. е. за вычетом простоев в ТОиР).

Целевое значение уровня технической готовности дробилки $\left(K_{\text {тг(д) }}\right)$ должно быть близким к ее коэффициенту внутренней готовности $\left(К_{\text {вг(д) }}\right)$. Он определяется исходя из нормированного ремонтного цикла, который устанавливается с учетом конструктивных и эксплуатационных особенностей оборудования, режима его работы и физико-механических свойств дробимых горных пород (табл. 2). На рис. 4 приведены значения К вг $_{\text {и }} \mathrm{K}_{\text {тг }}$ для основных дробилок крупного, среднего и мелкого дробления типоразмерной линейки ПАО «Уралмашзавод» при агрегатно-узловом методе ремонта. Коэффициенты готовности рассчитаны по реальным данным действующих ГОКов.

Несмотря на отклонения данных от строгих зависимостей, связанные с различными схемами цепи аппаратов дробильного передела, физикомеханическими свойствами руд, организацией ТОиР, по рис. 4 выделяются основные закономерности:

1) В стандартных условиях коэффициент технической готовности дробилки незначительно отличается от $\mathrm{K}_{\text {вг }}$, поскольку мала вероятность сверхнормативных нагрузок, попадания недробимых тел. В тяжелых условиях (рис. $4,6,6$ ) «просадка»

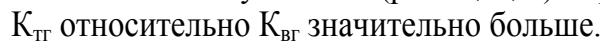

2) В наиболее жестких условиях работы по варианту II (прочность 200 МПа, абразивность 0,6-0,7,

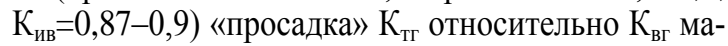
ла. Это объясняется частыми плановыми ремонтами, а потому минимальной вероятностью в эти короткие периоды внештатных ситуаций и неплановых ремонтов.

3) Коэффициент готовности в комплексе $\left(\mathrm{K}_{\text {тг(к) }}\right)$ наименьший у щековых дробилок, что связано с невозможностью их работы под завалом руды, а потому они всегда сопряжены с питателем, часто неподвижным (колосниковым) грохотом и др. Это вызывает дополнительные затраты времени на ТОиР, в том числе неплановые.

4) Дробилки среднего и мелкого дробления серии КСМД-2200 имеют больший диапазон изменения 
$\mathrm{K}_{\mathrm{тг}}$, что связано с высокой зависимостью от возникновения возможных сверхнормативных нагрузок (попадание недробимых тел) из-за малого размера и технологического назначения разгрузочной щели. В то же время влияние факторов связанности комплекса не столь существенно, так как дробилки чаще всего используются в параллельных технологических потоках по 4-12 шт. и остановка на ремонт одной из них практически не влияет на работу остальных.

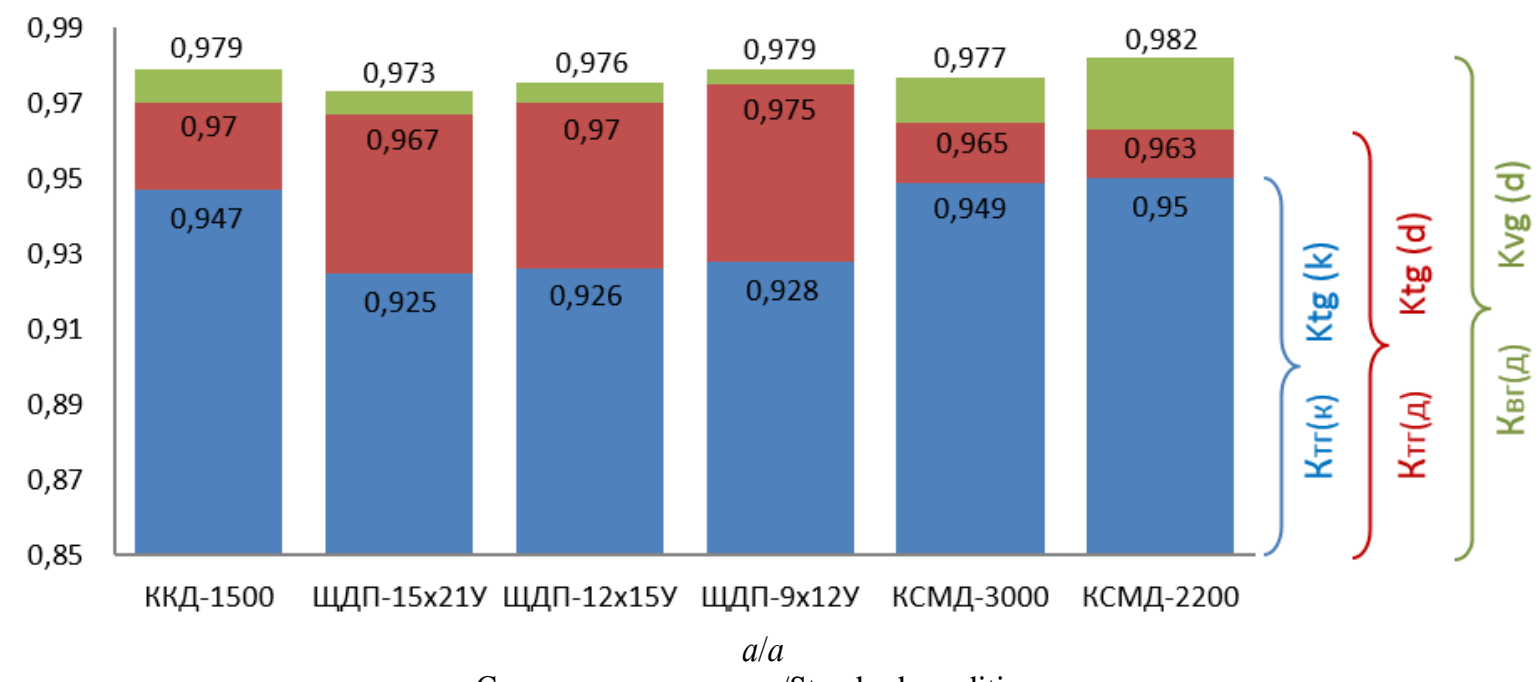

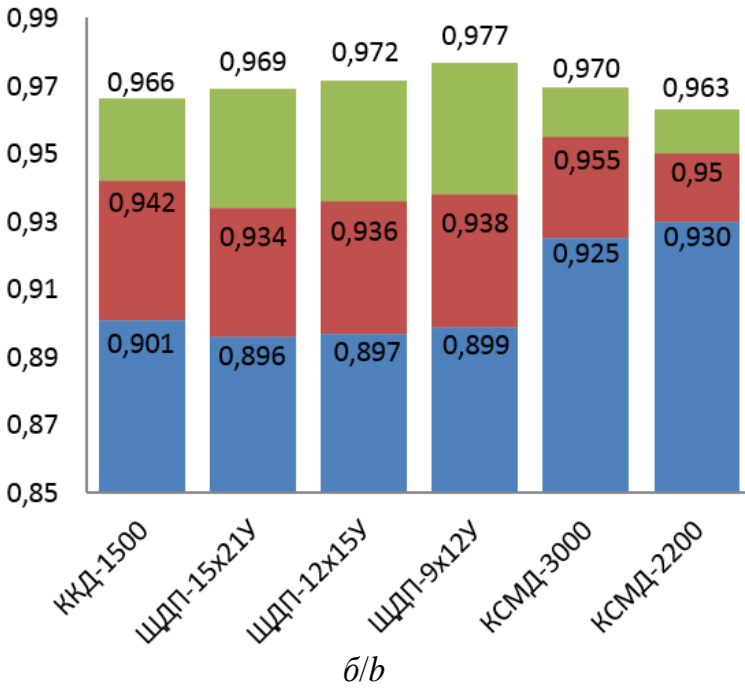

Крепкие абразивные руды Highly abrasive strong ores

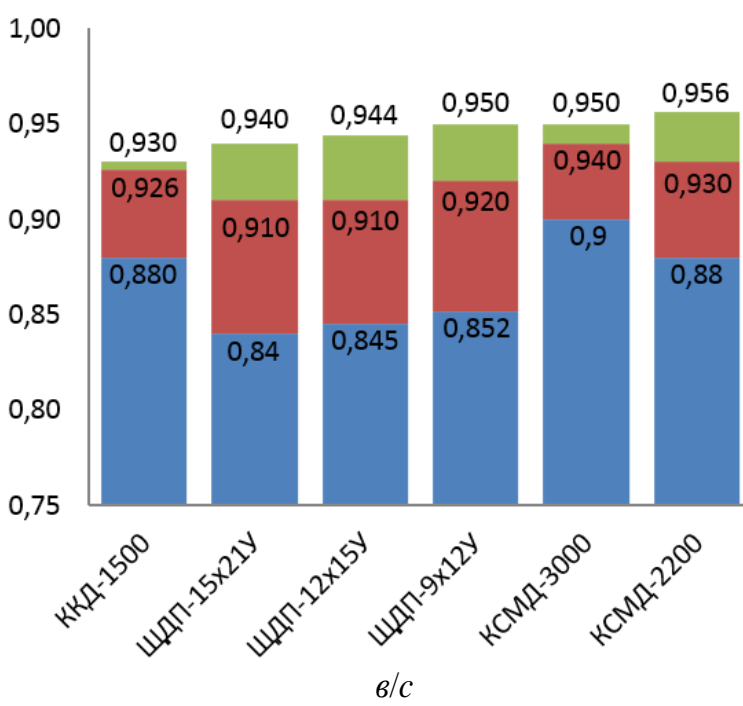

Высокопрочные высокоабразивные руды (II) Highly abrasive strong ores (II)

Pис. 4. Нормализованные значения коэффициентов готовности для разных моделей дробилок $\left(K_{\text {тә }(\kappa)}-\right.$ коэффициент технической готовности комплекса, $K_{\text {тг(д) }}$ - коэффициент технической готовности дробилки; Квг - коэффициент внутренней готовности дробилки)

Fig. 4. Normalized availability factors for different crusher models $\left(K_{t g(d)}, K_{t g(k)}, K_{v g(d)}-\right.$ technical readiness coefficients of the crusher and the «crusher + related equipment» complex, coefficient of internal ready crusher, respectively)

Анализ опыта эксплуатации дробилок позволяет сформулировать требования к организации их работы и порядку загрузки для обеспечения высокой производительности за счет максимального использования технических возможностей:

1. Оптимизировать систему технической эксплуатации (часто ее сводят к системе ТОиР, что ограничивает возможности управления параметрами системы) для достижения максимальных коэффициентов готовности. Целевая функция при оптимизации должна предполагать повышение коэффи-

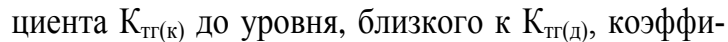

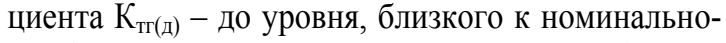

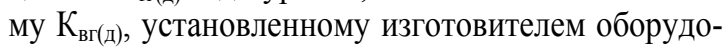
вания, а коэффициента $K_{\text {ив }}-$ к 1 :

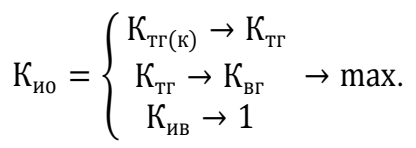

Это требует применения прогрессивных методов с переходом от планово-предупредительной системы к эффективной системе обеспечения работоспособности 
[18] с выстраиванием системы раннего выявления неисправностей и прогнозной аналитики на базе цифровых систем, системы непрерывного мониторинга качества применяемых запасных частей и материалов. Кроме этого, для повышения эффективности процесса рудоподготовки можно проводить мероприятия организационного и технологического характера при выполнении всех видов ремонтных воздействий (техническое обслуживание, текущие и капитальные ремонты) [19].

Таким образом, для выявления резервов совершенствования технической эксплуатации оборудования необходимо наряду с интегральным показателем - коэффициентом использования оборудования внедрять раздельный учет коэффициентов внутренней и технической готовности, а также коэффициента использования во времени.

2. Схема загрузки приемного бункера должна обеспечивать заданную часовую интенсивность загрузки с учетом грузоподъемности транспортных средств и равномерности их подачи. Для современных и перспективных конусных дробилок крупного дробления с производительностью 5000-7000 т/ч рекомендуется обеспечить минимум два места для одновременной разгрузки транспорта в каждую дробилку, при этом грузоподъемность транспорта - не менее 115-130 т на один транспортный сосуд, либо погрузчик с ковшом не менее $15-17 \mathrm{~m}^{3}$.

3. Обеспечить равномерность подачи транспорта на разгрузку (коэффициент равномерности не ниже $0,8)$. Эта задача может решаться за счет оптимизирующих автоматизированных систем диспетчеризации [20] совместно с повышением производительности системы выемочно-погрузочного и транспортного оборудования.

4. При невозможности достижения высокой равномерности подачи транспорта необходимо увеличивать производительность дробилки (форсировать ее) путем модернизации под требуемую часовую производительность $[16,21]$.

\section{Выводы}

1. В условиях необходимости наращивания производственной мощности действующих горнообогатительных комбинатов важным и реализуемым является использование резервов повышения производительности действующего дробильного оборудования. Приведенные расчеты позволили

\section{СПИСОК ЛИТЕРАТУРЬ}

1. Схемы циклично-поточной технологии при различном залегании месторождений полезных ископаемых (обзор применяемых схем ЦПТ) / В.А. Бурсенев, Г.Д. Кармаев, А.В. Семенкин, И.Г. Сумина // Проблемы недропользования. - 2018. № 4 (19). - C. 13-21. DOI: 10.25635/2313-1586.2018.04.013

2. Проблемы магистрального транспортирования руды от удаленных кимберлитовых месторождений / П.И. Тарасов, А.Г. Журавлев, В.А. Черепанов, М.В. Исаков, В.Р. Баланчук, А.Н. Акишев, С.Л. Бабаскин // Горное оборудование и электромеханика. - 2014. - № 5. - С. 25-31.

3. Зырянов И.В., Кондратюк А.П. Рациональная конфигурация многозвенных автопоездов для транспортировки кимберлито- установить обоснованные величины этих резервов: 10-30 \% за счет обеспечения равномерной подачи транспорта на разгрузку, 5-10 \% за счет оптимизации порядка работы цепи аппаратов ДОФ во времени, 3-5 \% за счет совершенствования технического обслуживания и ремонта.

2. Для стабильной высокопроизводительной работы транспортно-обогатительного передела горного предприятия необходимо одновременно налаживать организацию взаимодействия транспортного и дробильного оборудования и формировать эффективную систему обеспечения работоспособности этого оборудования, в том числе за счет максимального использования технических возможностей:

- оптимизировать систему технической эксплуатации дробильного оборудования для достижения максимальных коэффициентов готовности, в том числе за счет внедрения раздельного учета коэффициентов внутренней и технической готовности, а также коэффициента использования во времени;

- схема загрузки приемного бункера и применяемое дробильное оборудование должны обеспечивать заданную часовую интенсивность загрузки с учетом грузоподъемности транспортных средств и равномерности их подачи;

- обеспечить равномерность подачи транспорта на разгрузку (коэффициент равномерности не ниже 0,8$)$.

3. Одним их значимых инструментов повышения производительности и эффективности транспортной системы карьера и дробильного передела является внедрение цифровых систем прогнозной аналитики и оптимизированного управления.

4. Для дробилок среднего и мелкого дробления проблема попадания недробимых тел - это один из ключевых негативных факторов, вызывающий либо нарушение работоспособности дробилок, либо простои линии во время удаления недробимых тел с ленты конвейера при их своевременном обнаружении. Следовательно, остро стоит проблема разработки эффективных систем быстрого детектирования и удаления из потока недробимых тел (прежде всего металлических).

Исследования выполнены в рамках Государственного задания № ГР ААAA-A19-119020790025-4.

вой породы АК «Алроса» // Горный информационноаналитический бюллетень. - 2017. - № S24. - С. 229-239.

4. Features of modern approach to selection of haulage systems for open pit diamond mines in Yakutia / V.L. Yakovlev, I.V. Zyryanov, A.G. Zhuravlev, V.A. Cherepanov // Journal of Mining Science. - 2018. - V. 54. - № 6. - P. 979-987. DOI: 10.1134/S1062739118065131

5. Barabady J., Kumar U. Reliability analysis of mining equipment: a case study of a crushing plant at Jajarm Bauxite Mine in Iran // Reliability Engineering and System Safety. - 2008. - V. 93. Iss. 4. - P. 647-653

6. Barabady J. Reliability and maintainability analysis of crushing plants in Jajarm Bauxite Mine of Iran // Annual Reliability and Maintainability Symposium, International Symposium on Product 
Quality and Integrity. - Alexandria, Virginia, USA, 2005, January 24-27. - Piscataway, NJ: IEEE Communications Society, 2005. P. $109-115$.

7. Availability based simulation and optimization modeling framework for open-pit mine truck allocation under dynamic constraints / R. Mena, E. Zio, F. Kristjanpoller, A. Arata // International Journal of Mining Science and Technology. 2013. - V. 23. - № 1. - P. 113-119.

8. Tsai Y.T., Wang K.S., Tasi L.C. A study of availability-centered preventive maintenance for multi-component systems // Reliability Engineering \& System Safety. - 2004 - - V. 84. - № 3 - P. 261-270.

9. Hall R.A., Daneshmend L.K. Reliability modeling of surface mining equipment: data gathering and analysis methodologies // International Journal of Surface Mining, Reclamation and Environment. - 2003. - V. 17. - № 3. - P. 139-155.

10. Ghodrati B., Kumar U. Reliability and operating environmentbased spare parts estimation approach: a case study in Kiruna Mine, Sweden // Journal of Quality in Maintenance Engineering. 2005. - V. 11. - № 2. - P. 169-184.

11. Dhillon B.S., Anude O.C., Mining equipment reliability: a review // Microelectronics and Reliability. - 1992. - V. 32. - № 8. P. 1137-1156.

12. Lewis M.W., Steinberg L. Maintenance of mobile mine equipment in the information age // Journal of Quality in Maintenance Engineering. - 2001. - V. 7. - № 4. - P. 264-274.

13. Усов С.В., Белов С.В. Повышение коэффициента технической готовности погрузочно-доставочного комплекса карьера // Технологическое оборудование для горной и нефтегазовой промышленности: сборник трудов XVI международной научно-технической конференции «Чтения памяти В.Р. Кубачека», проведенной в рамках Уральской горонопромышленной декады 12-13 апреля 2018 г. - Екатеринбург: Уральский государственный горный университет, 2018. - С. 350-352.

14. Лагунова Ю.А., Брусова О.М. Влияние коэффициента готовности на структуру ремонтного цикла дробильного оборудо- вания // Горный информационно-аналитический бюллетень. 2012. - № S1-1. - C. 102-107.

15. ГОСТ 27.002-2015. Надежность в технике (ССНТ). Термины и определения. - М.: Стандартинформ, 2016. -39 с

16. Стойленский ГОК и УЗТМ открыли новую эпоху в сегменте дробления руды. URL: https://uralmash-kartex.ru/stojlenskij-goki-uztm-otkryili-novuyu-epoxu-v-segmente-drobleniya-rudyi (дата обращения 30.04 .2020 ).

17. Методический подход к разработке регламентов технического обслуживания и ремонта дробильного оборудования горнодобывающих предприятий / А.Г. Журавлев, М.А. Чендырев, И.А. Глебов, В.А. Черепанов // Горный информационноаналитический бюллетень. - 2020. - № 3-1. - С. 543-556. DOI: 10.25018/0236-1493-2020-31-0-543-556.

18. Андреева Л.И., Красникова Т.И., Ушаков Ю.Ю. Методология формирования эффективной системы обеспечения работоспособности горной техники // Известия вузов. Горный журнал. 2019. - № 5. - С. 92-106.

19. Андреева Л.И. Оценка резервов повышения эффективности процесса рудоподготовки в АО «Ковдорский ГОК» // Горный информационно-аналитический бюллетень. - 2019. - № 1. C. 185-192. DOI: 10.25018/0236-1493-2019-01-0-185-192

20. Журавлев А.Г., Ченцов П.А. Возможности автоматизированной оптимизации работы транспортных систем карьеров на основе мультиагентного подхода // Горный информационноаналитический бюллетень. - 2019. - № 11 (СЦ 37). - С. 141-150. DOI: 10.25018/0236-1493-2019-11-37-141-150.

21. Лагунова Ю.А., Фурин В.О., Федулов К.А. Конусные дробилки ПАО «Уралмашзавод» для конкретных условий эксплуатации // Горное оборудование и электромеханика. - 2018. № 1 (135). - C. $27-33$.

Поступила 22.10.2020 2.

\section{Информация об авторах}

Журавлев А.Г., кандидат технических наук, заведующий лабораторией, Институт горного дела УрО РАН.

Черепанов В.А., научный сотрудник, начальник лаборатории неразрушающего контроля, Институт горного дела УрО РАН. 


\title{
RESERVES TO INCREASE PRODUCTIVITY OF «QUARRY TRANSPORT - CRUSHING-AND-CONCENTRATING PLANT» SUBSYSTEM TAKING INTO ACCOUNT CRUSHING EQUIPMENT UTILIZATION FACTORS
}

\author{
Artem G. Zhuravlev', \\ juravlev@igduran.ru \\ Vladimir A. Cherepanov ${ }^{1}$, \\ transport@igduran.ru \\ 1 Institute of Mining of Ural branch of RAS, \\ 58, Mamin-Sibiryak street, Ekaterinburg, 620219, Russia.
}

\begin{abstract}
The relevance. Under current conditions, when it is necessary to increase the production capacity of existing mining-and-processing integrated works, the growth in productivity of the quarry cannot be considered in isolation from the capabilities of crushing and processing mills. When solving issues of increasing the capacity of quarry transport, it is necessary to pay attention to optimization of its interaction with the primary stages of enrichment production, and for this purpose it is necessary to consider it together with the link of main transport as a single technological system.

The main aim of the study is to identify reserves for increasing the productivity of the subsystem enterprise "quarry transport - crushingand-concentrating plant» taking into account the utilization factors of crushing equipment based on a synthesis of experience in its operation and the requirements for operational reliability prevailing in modern conditions.

Objects: quarry transport and crushing equipment

Methods: motion-time study, statistical data processing, systematization, analysis and generalization, performance calculation

Results. The most common technological schemes of transport and crushing equipment used at modern mining and processing plants are structured. The key limiting factors for increasing productivity for the subsystem of the mining enterprise "quarry transport - crushing-andconcentrating plant» are identified. Calculations of the achievable loading intensity by various mining vehicles for stable high-performance crushers work were made. The reliability indicators of crushers are determined and calculated. The main factors affecting their operational reliability, the required frequency and amount of repair work, as well as the time of productive work are identified. An analysis of the operating experience of crushers is carried out and the requirements for the organization of their work and the loading order are formulated to ensure high performance. In conditions of necessity to increase production capacity of existing mining and processing plants, it is important and feasible to use reserves to increase productivity of existing crushing equipment: $10-30 \%$ due to provision of uniform supply of transport for unloading, 5-10\% due to optimization of operation procedure of the crushing and processing plant circuit in time, 3-5 \% due to improvement of maintenance and repair. At the same time, the increase in the utilization factor of equipment of crushing and enrichment factories should be considered in relation to operation of technological quarry transport. For the stable high-performance work of transportation and processing facilities of a mining company, it is necessary to simultaneously organize the interaction between transport and crushing equipment and form an effective system to ensure the operability of this equipment, including by maximizing the use of technical capabilities.
\end{abstract}

\section{Key words:}

Quarry transport, crusher, receiving bin, availability rate, efficiency, productivity, crushing equipment.

The study was carried out according to the State Task no. GR AAAA-A19-119020790025-4.

\section{REFERENCES}

1. Bersenev V.A., Karmaev D.G., Semenkin A.V., Sumina I.G. Schemes of cyclic-flow technology by various bedding of mineral deposits (reviewing of existing and proposed schemes of CFT). Problemy nedropolzovaniya, 2018, no. 4, pp. 13-21. In Rus. DOI: 10.25635/2313-1586.2018.04.013.

2. Tarasov P.I., Zhuravlev A.G., Cherepanov V.A., Isakov M.V., Balanchuk V.R., Akishev A.N., Babaskin S.L. Problemy magistralnogo transportirovaniya rudy ot udalennykh kimberlitovykh mestorozhdeniy [Mainline ore transportation problems on the remote kimberlite fields]. Gornoe oborudovanie i elektromekhanika, 2014, no. 5, pp. 25-31.

3. Zyryanov I.V., Kondratyuk A.P. Rational configuration of multilink trucks for transportation kimberlite rocks of AK ALROSA MIAB. Mining Inf. Anal. Bull., 2017, no. S24, pp. 229-239. In Rus.

4. Yakovlev V.L., Zyryanov I.V., Zhuravlev A.G., Cherepanov V.A. Features of modern approach to selection of haulage systems for open pit diamond mines in Yakutia. Journal of Mining Science, 2018, vol. 54, no. 6, pp. 979-987. DOI: 10.1134/ S1062739118065131.

5. Barabady J., Kumar U. Reliability analysis of mining equipment: a case study of a crushing plant at Jajarm Bauxite Mine in Iran. Re- liability Engineering and System Safety, 2008, vol. 93, Iss. 4, pp. 647-653.

6. Barabady J. Reliability and maintainability analysis of crushing plants in Jajarm Bauxite Mine of Iran. Annual Reliability and Maintainability Symposium, International Symposium on Product Quality and Integrity. Alexandria, Virginia, USA, 2005, January 24-27. Piscataway, NJ, IEEE Communications Society, 2005. pp. 109-115.

7. Mena R., Zio E., Kristjanpoller F., Arata A. Availability based simulation and optimization modeling framework for open-pit mine truck allocation under dynamic constraints. International Journal of Mining Science and Technology, 2013, vol. 23, no. 1, pp. 113-119.

8. Tsai Y.T., Wang K.S., Tasi L.C. A study of availability-centered preventive maintenance for multi-component systems. Reliability Engineering \& System Safety, 2004, vol. 84, no. 3, pp. 261-270.

9. Hall R.A., Daneshmend L.K. Reliability modeling of surface mining equipment: data gathering and analysis methodologies. International Journal of Surface Mining, Reclamation and Environment, 2003, vol. 17, no. 3, pp. 139-155.

10. Ghodrati B., Kumar U. Reliability and operating environmentbased spare parts estimation approach: a case study in Kiruna 
Mine, Sweden. Journal of Quality in Maintenance Engineering, 2005, vol. 11, no. 2, pp. 169-184.

11. Dhillon B.S., Anude O.C. Mining equipment reliability: a review. Microelectronics and Reliability, 1992, vol. 32, no. 8, pp. 1137-1156.

12. Lewis M.W., Steinberg L. Maintenance of mobile mine equipment in the information age. Journal of Quality in Maintenance Engineering, 2001, vol. 7, no. 4, pp. 264-274.

13. Usov S.V., Belov S.V. Povyshenie koeffitsienta tekhnicheskoy gotovnosti pogruzochno-dostavochnogo kompleksa karera [Increase of the coefficient of technical readiness for loading and delivery of complex career]. Tekhnologicheskoe oborudovanie dlya gornoy $i$ neftegazovoy promyshlennosti. Sbornik trudov XVI mezhdunarodnoy nauchno-tekhnicheskoy konferentsii «Chteniya pamyati V.R. Kubacheka» [Technological equipment for the mining and oil and gas industries. Proc. of the XVI international scientific and technical conference «Readings in Memory of V.R. Kubachek»]. Yekaterinburg, Ural State Mining University Publ., 2018. pp. 350-352.

14. Lagunova Yu.A., Brusova O.M. Influence on the structure of coefficient of readiness to repair cycle of crushing equipment. $M I$ AB. Mining Inf. Anal. Bull., 2012, no. S1-1, pp. 102-107. In Rus.

15. GOST 27.002-2015. Nadezhnost $v$ tekhnike (SSNT). Terminy i opredeleniya [State Standard 27.002-2015. Reliability in technology. Terms and Definitions]. Moscow, Standartinform Publ., 2016. 39 p.

16. Stoylenskiy GOK i UZTM otkryli novuyu epokhu v segmente drobleniya rudy [Stoilensky Mining and Beneficiation Plant and Uralmashplant ushered in a new era in the ore crushing segment]. Available at: https://uralmash-kartex.ru/stojlenskij-gok-i-uztm- otkryili-novuyu-epoxu-v-segmente-drobleniya-rudyi (accessed 30 April 2020).

17. Zhuravlev A.G., Chendyrev M.A., Cherepanov V.A., Glebov I.A. Methodical approach to the development of regulations of maintenance and repair of crushing equipment of mining companies. $M I$ AB. Mining Inf. Anal. Bull., 2020, no. 3-1, pp. 543-556. In Rus. DOI: 10.25018/0236- 1493-2020-31-0-543-556.

18. Andreeva L.I., Krasnikova T.I., Ushakov Yu.Yu. The methodology for the formation of an effective system to ensure the performance of mining equipment. News of the Higher Institutions. Mining Journal, 2019, no. 5, pp. 92-106. In Rus. DOI: 10.21440/0536-1028-2019-5-92-106.

19. Andreeva L.I. Assessment of efficiency improvement potentiality in ore pretreatment at Kovdor Mining and Processing Plant. MIAB. Mining Inf. Anal. Bull., 2019, no. 1, pp. 185-192. In Rus. DOI: 10.25018/0236-1493-2019-01-0-185-192.

20. Zhuravlev A.G., Chentsov P.A. Possibilities of automated optimization of the operation of quarry transport systems based on a multi-agent approach. MIAB. Mining Inf. Anal. Bull., 2019, no. S11, pp. 141-150. In Rus. DOI: 10.25018/0236-1493-2019-11-37-141-150,

21. Lagunova Yu.A., Furin V.O., Fedulov K.A. Konusnye drobilki PAO «Uralmashzavod» dlya konkretnykh usloviy ekspluatatsii [Cone crushers of PJSC «Uralmashzavod» for specific operating conditions]. Gornoe oborudovanie i elektromekhanika, 2018, no. 1 (135), pp. 27-33.

Received: 22 October 2020

\section{Information about the authors}

Artem G. Zhuravlev, Cand. Sc., assistant professor, laboratory chief, Institute of Mining of Ural branch of RAS.

Vladimir A. Cherepanov, researcher, head of the nondestructive testing laboratory, Institute of Mining of Ural branch of RAS. 